\title{
Bilateral simultaneous acute angle closure in an adult Nepalese woman
}

\author{
Sabin Sahu ${ }^{1}$, Lila Raj Puri ${ }^{1}$ \\ 'Sagarmatha Choudhary Eye Hospital, Lahan, Nepal
}

\begin{abstract}
Purpose: To report a case of bilateral simultaneous angle closure in an adult Nepalese woman without any known secondary cause.

Methods: Observational case report.

Results: A 50-year-old Nepalese woman presented with decreased vision, pain, redness, and watering in both eyes with associated coloured haloes, nausea, and vomiting for 10 days. At presentation, her visual acuity was 20/400 in the right eye and hand motions close to face with accurate projection of rays in the left eye. Intraocular pressures was $38 \mathrm{mmHg}$ in the right eye and $48 \mathrm{mmHg}$ in the left eye without any antiglaucoma medications. $A$ slit-lamp examination revealed bilateral circum-corneal conjunctival congestion, corneal edema, and shallow anterior chambers. Both pupils were mid-dilated and non-reactive to light. Gonioscopy showed closed angles in all four quadrants bilaterally. Posterior segment examination revealed normal optic disc with cup-disc-ratio of 0.3 in the right eye, and blurring of disc margin with cup-disc-ratio of 0.3 in the left eye. The patient was started on systemic acetazolamide $250 \mathrm{mg} 4$ times a day, topical brimonidine $0.2 \%$ and timolol $0.5 \% 2$ times a day, and topical dexamethasone 6 times a day in both eyes, following which IOP reduced to 11 and $12 \mathrm{mmHg}$, respectively, the corneal edema subsided, but the anterior chamber remained shallow. The Laser peripheral iridotomy was performed in the right eye and surgical peripheral iridectomy was performed in the left eye. After two weeks, vision improved to 20/30 in both eyes with normal intraocular pressures off antiglaucoma medications. Anterior chambers deepened significantly with clear corneas bilaterally. Gonioscopy at this stage showed essentially open angles with appositional closure in superior and temporal quadrants in the right eye and open angles in all four quadrants in the left eye. Posterior segment evaluation revealed normal optic disc in both eyes.

Conclusions: Bilateral simultaneous acute angle closure is a rare presentation with very few reported secondary causes. We report a case of bilateral simultaneous angle closure in an adult Nepalese woman without any known secondary cause. The case was successfully managed with laser peripheral iridotomy in the right eye and surgical peripheral iridectomy in the left eye.
\end{abstract}

Keywords: acute angle closure, bilateral simultaneous, secondary cause

\section{Introduction}

Bilateral acute angle closure is a rare entity and has been reported secondary to drugs $^{1}$, general anesthesia ${ }^{2}$, snake bite ${ }^{3}$, microspherophakia ${ }^{4}$ and as an initial symptom of Vogt-Koyanagi-Harada syndrome. ${ }^{5}$ Among drugs, bilateral angle closure has been reported secondary to topiramate, a commonly used antimigraine

Correspondence: Sabin Sahu, Ophthalmologist, Sagarmatha Choudhary Eye Hospital, Lahan - 3, Siraha, Nepal.

E mail: sabinsahu@gmail.com 
and antiepileptic drug ${ }^{6,7,8}$ and sulphonamides. ${ }^{9}$ We report a case of bilateral simultaneous angle closure in a middle-aged Nepalese woman without any known secondary cause.

\section{Case report}

A 50-year-old Nepalese woman presented to the OPD with decreased vision, pain, redness, and watering in both eyes with associated coloured haloes, nausea, and vomiting for 10 days. She noticed the symptoms first in her left eye in the morning while she was doing her normal daily activities, followed by similar symptoms in the right eye within few hours. There was no previous history of such episodes. At presentation, her visual acuity was 20/400 in right eye and hand motions close to face with accurate projection of rays in left eye. Intraocular pressures was $38 \mathrm{mmHg}$ in the right eye and $48 \mathrm{mmHg}$ in the left eye without any antiglaucoma medications. A slit-lamp examination revealed bilateral circum-corneal conjunctival congestion, corneal edema, and shallow anterior chambers (Fig. 1). Both pupils were mid-dilated and non-reactive to light. The lens showed early posterior subcapsular cataract in the right eye while the left eye showed early posterior subcapsular cataract with glaukomflecken. There was cellular reaction $2+$ in the right eye and $3+$ in the left eye. Posterior segment examination of the right eye revealed a normal optic disc with vertical cup-disc ratio of 0.3 , while the left eye revealed blurring of the disc margin with vertical cup-disc ratio of 0.3 . Gonioscopy showed closed angles in all four quadrants in both eyes. The clinical diagnosis of bilateral acute angle closure was made. There was no history suggestive of any of the reported causes of bilateral acute angle closure like drug intake, general anesthesia, or snake bite.

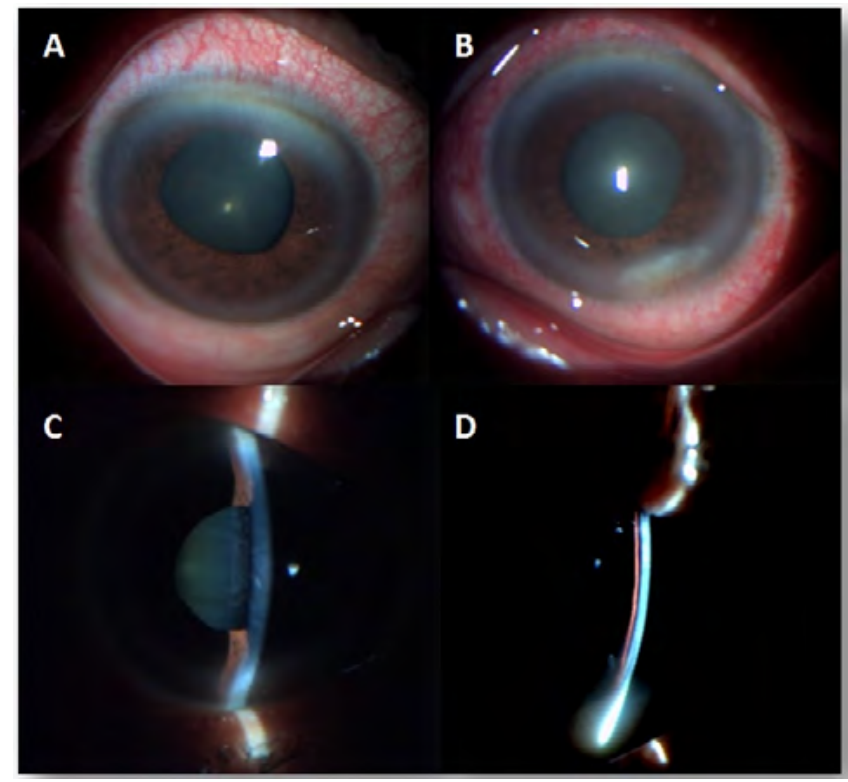

Fig 1. A slit-lamp photograph of the patient's right eye $(A)$ and left eye (B) showing conjunctival congestion, corneal haze and fixed mid-dilated pupil. Slit section showing shallow central (C) and peripheral (D) anterior chamber. 
The patient was started on systemic acetazolamide $250 \mathrm{mg} 4$ times a day, topical brimonidine $0.2 \%$ and timolol $0.5 \%$ combination 2 times a day and topical steroid dexamethasone 6 times a day in both eyes.

On follow-up the next day, the anterior chamber remained shallow, but the IOP reduced to 11 and $12 \mathrm{mmHg}$, respectively, the corneal edema had subsided, and the patient was symptomatically better. Retinoscopy showed a refractive error of +1.0 DS/-1.0 DC $\times 90^{\circ}$ in the right eye and +1.0 DS/-1.5 DC x 90 in the left eye. The Laser peripheral iridotomy was performed in the right eye. However, laser iridotomy could not be performed in the left eye due to poor visibility in the periphery; surgical peripheral iridectomy was performed instead. The antiglaucoma medications were stopped and the topical steroid was continued postoperatively in tapering doses.

On two weeks follow-up, vision improved to 20/30 with -1.0 DS correction in the right eye and $20 / 30$ with $-0.75 \mathrm{DC} \times 100^{\circ}$ correction in the left eye. Intraocular pressure was 18 and $12 \mathrm{mmHg}$ off drugs in the right and left eye, respectively. Anterior chambers were quiet with clear corneas in both eyes. Both the laser PI in right eye and the surgical PI in the left eye were patent with significantly deepened anterior chambers postoperatively in both eyes (Fig. 2). Gonioscopy at this stage showed essentially open angles with appositional closure in superior and temporal quadrants in right eye and open angles in all four quadrants in the left eye. Posterior segment evaluation revealed normal optic disc in both eyes. We continue to monitor her closely.

\section{Discussion}

Angle closure is more common in older age, female sex, and the Asian population due to the anatomic risk factor of shallow anterior chambers and a shorter axial length of the globe. ${ }^{10}$ The acute presentation of the angle closure is common, most of them being the unilateral presentation. In a prospective study of incidence of acute angle closure in Singapore, the annual incidence rate of acute angle closure
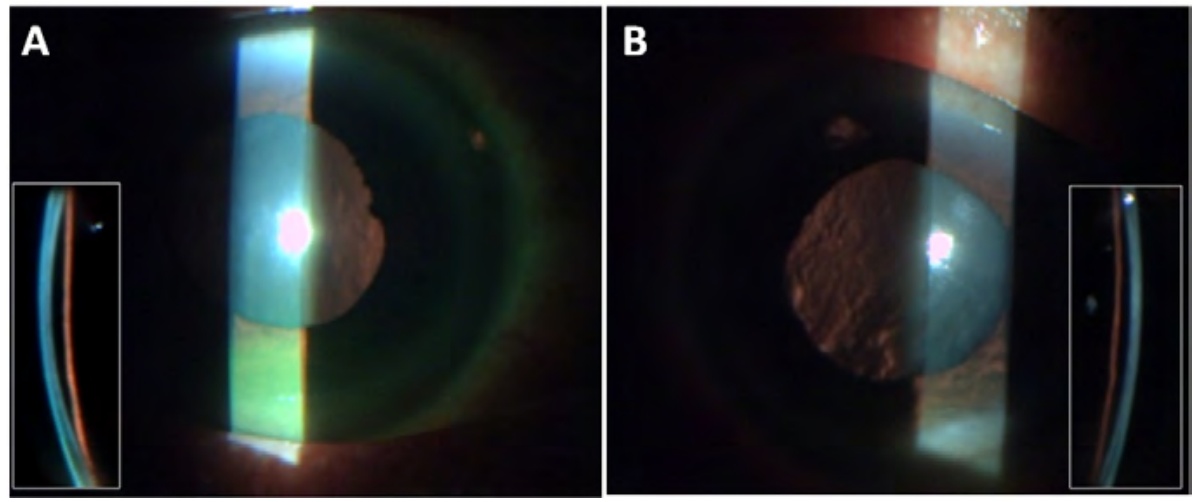

Fig 2. A slit-lamp photograph of the patient's right eye $(A)$ showing the patent laser peripheral iridotomy with deepened anterior chamber postoperatively (offset) and left eye (B) showing the patent surgical iridectomy with deepened anterior chamber postoperatively (offset). 
was 12.2 per $1,00,000$ in those aged 30 years and older. ${ }^{11}$ Bilateral simultaneous angle closure is, however, a rare presentation. It has been reported to occur secondary to drugs like topiramate ${ }^{6,7,8}$ and sulphonamides ${ }^{9}$, general anesthesia ${ }^{2}$, snake bite ${ }^{3}$, microspherophakia ${ }^{4}$ and as an initial symptom of Vogt-KoyanagiHarada syndrome. ${ }^{5}$ Our case is unique since there is no known secondary cause for the bilateral simultaneous acute angle closure. The cellular reactions are usually mild to moderate in the anterior chamber in acute angle closure attack, but in this case the cellular reaction was intense probably because of severe or prolonged attack. The case was successfully managed with laser peripheral iridotomy in the right eye and surgical peripheral iridectomy in the left eye. Laser peripheral iridotomy was attempted first in the left eye as well, but we failed to produce patent iridotomy, so surgical peripheral iridectomy was performed instead. After the surgical intervention, we were able to document her vision after two weeks only since she resided in a remote hilly place in Nepal which was far from our hospital. However, she may have recovered her vision much earlier. The pupils were still mid-dilated after normalization of intraocular pressure and patent peripheral iridotomies when we examined her at two weeks time probably because of the iris sphincter muscle ischemia, which may have occurred due to prolonged raised intraocular pressure due to the late presentation of the patient.

\section{Acknowledgements}

We would like to thank Reena Yadav MD, and Kabina Shrestha MD for helping in management of the case and Dipesh Ram for the photography.

\section{References}

1. Tripathi RC, Tripathi JB, Haggerty C. Drug Induced glaucomas: mechanism and management. Drug Saf.2003; 26: 749-67.

2. Ates $\mathrm{H}$, Kayikcioglu $\mathrm{O}$, Andac K. Bilateral angle closure glaucoma following general anesthesia. Int Ophthalmol. 1999; 23: 129-30.

3. Srinivasan R, Kaliaperumal S, Dutta TK. Bilateral angle closure glaucoma following snake bite. J Assoc Physicians India. 2005; 53: 46-48.

4. Kaushik S, Sachdev N, Pandav SS, Gupta A, Ram J. Bilateral acute angle closure glaucoma as a presentation of isolated microspherophakia in an adult: case report. BMC Ophthalmol. 2006; 6:29.

5. Kimura R, Sakai M, Otabe H. Transient shallow anterior chamber as initial symptom in Harada's syndrome. Arch Ophthalmol. 1981; 99: 1604-6.

6. Levy J, Yagev R, Petrova A, Lifshitz T. Topiramate-induced bilateral angle-closure glaucoma. Can J Ophthalmol.2006; 41: 221-5.

7. Cruciani F, Lorenzatti M, Nazzarro V, Abdolrahimzadeh S. Bilateral acute angle closure glaucoma and myopia induced by topiramate. Clin Ter. 2009; 160: 215-6.

8. Grewal DS, Goldstein DA, Khatana AK, Tanna AP. Bilateral angle closure following use of a weight loss combination agent containing topiramate. J Glaucoma. 2015; 24: 132-6.

9. Lee GC, Tam CP, Danesh-Meyer HV, Myers JS, Katz LJ. Bilateral angle closure glaucoma induced by sulphonamide-derived medications. Clin Experiment Ophthalmol. 2007; 35: 55-8.

10. Foster PJ. The epidemiology of primary angle closure and associated glaucomatous optic neuropathy. Semin Ophthalmol. 2002 Jun; 17: 50-8.

11. Seah SK, Foster PJ, Chew PT, Jap A, Oen F, Fam HB, Lim AS. Incidence of acute primary angle closure glaucoma in Singapore. An island-wide survey. Arch Ophthalmol. 1997; 115: 1436-40. 